\title{
MEMBRANE INTERFACE PROBE (MIP) - INNOVATIVE APPROACH TO INVESTIGATION AND REMEDIATION OF CONTAMINATED SITES
}

\author{
Vladislav Knyt| ${ }^{1}$, Jan Kukacka ${ }^{1}$, Ondrej Lhotsky ${ }^{1}$, Robert Raschman ${ }^{1}$ \\ 1 DEKONTA, a.s., Volutova 2523, 158 No0 Prague, Czech Republic, \\ info@dekonta.cz
}

\begin{abstract}
:
Membrane Interface Probe - MIP (Geoprobe, USA) is a helpful tool used to detect the presence of volatile organic compounds (VOCs) with depth in contaminated soil. Dekonta used MIP technology within several research projects. Further, Dekonta was participating on development of specially designed injection equipment (MIP-IN) complementary to MIP for efficient application of remediation agents.
\end{abstract}

Keywords: MIP, remediation, contaminated site, direct-push, investigation, ISCO

\section{Introduction}

Detailed investigation of the pollution of soils is an important part of contaminated sites clean-up. An important approach that can be used in remediation field represent direct sensing methods (eg. direct push methods). Most of these technologies are essential part of High Resolution Site Characterization (HSCR). This is a strategy developed by US Environmental Protection Agency (EPA) focusing on scale-appropriate measurement and sample density to define contaminant distributions, and the physical context in which they reside, with greater certainty, supporting faster and more effective site cleanup [1].

Among the most widespread technologies include Membrane Interface Probe (MIP) developed by Geoprobe company (Geoprobe, USA). MIP is a helpful tool used for detection of volatile organic compounds (VOCs) with depth in contaminated soil. MIP was used within many research projects for site characterisation (e.g. [2], [3]).

\section{Materials and Methods}

The MIP system is composed of a penetration probe, a control unit, and a gas chromatograph. Use of the technology on site consists in penetration of the probe into the soil using the drilling rig and continuous measurement of the gases sucked by the probe. The probe is heated during the whole period of penetration, and mobilised contaminants enter the system, on the principle of diffusion through a special semipermeable membrane, and then are carried by carrier gas - $\mathrm{N}_{2}$ to the GC. Further, the probe is equipped with a dipole sensor for measuring electrical conductivity (EC) of the surrounding environment, which helps to improve information on lithology profile (higher values of EC indicates low permeable formations). MIP may be used for a broad spectrum of volatile organic contaminants (BTEX, chlorinated hydrocarbons, petroleum substances, MTBE, etc.) The gas chromatograph is equipped with three detectors: PID (aromatic hydrocarbons, unsaturated hydrocarbons); FID (ethenes, light hydrocarbons C1-C9) and XSD (chlorinated hydrocarbons). The MIP assembly and example of log can be seen on following picture. 
$\mathbf{A}$

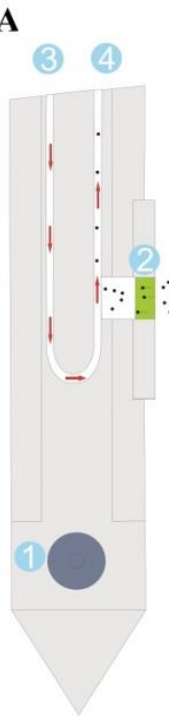

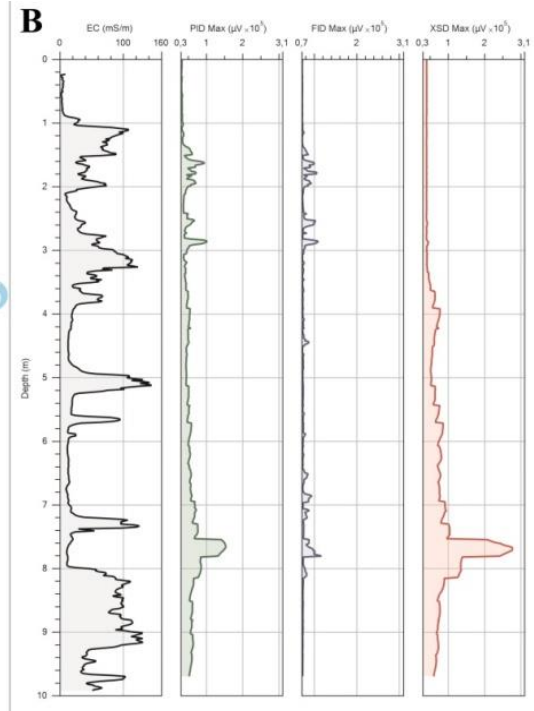

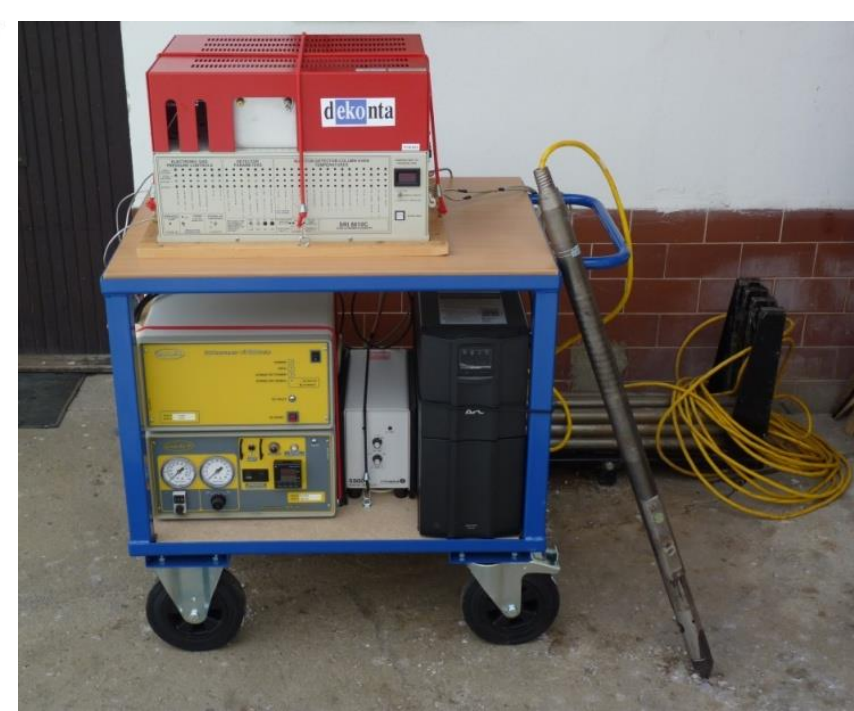

Figure 11: A) Scheme of MIP probe: 1) : 1) dipole for EC measurement, 2) semi-permeable membrane, 3) Teflon trunkline with sweeping carrier gas (N2), 4) PEEK trunkline, 5) contamination (VOCs)

B) An example of log; $1 . \log =\mathrm{EC}$ with clearly visible lower/higher permeable layers; 2.-4. $\log =$ signals from 3 detectors (PID, FID, XSD) installed inside GC

C) Portable MIP assembly

Thanks to the MIP technology, there can be found easily in which layers within the soil contamination is present. This approach is more flexible, quicker, and more precise, in comparison with the common methods (sampling of cores from plastic tubes during core drilling and their analytical determination in laboratory). In the case of more extensive contamination mapping, this causes significant time and financial savings. Moreover, exact information on spatial occurrence of contamination makes remediation solutions easier and more efficient. System of injection wells or application probes may be designed more precisely, and, thus, in-situ remediation technologies (chemical oxidation, chemical reduction, pump and treat, etc.) may be used more efficiently.

MIP is a semi-quantitative method and does not provide direct information about the concentration of pollutants in the soil or water. However, based on the signal intensity from the detector (unit $\mathrm{V}$ or $\mathrm{mV}$ ), it is possible to estimate the intensity of contamination. Afterwards it is possible to perform sampling of groundwater or soil in selected depth levels and correlate the intensity of the signal with the concentration of particular contaminant in groundwater or soil.

Obtained data may be used for preparing various graphic outputs in the form of contamination maps, 3D models, etc.

\section{Results and Discussion}

Dekonta company is carrying out both research and commercial activities at contaminated sites with complex contamination by various volatile organic compounds. Below we introduce examples of the use of MIP technology within several research projects. 


\section{Project \#1}

A good example is the Site \#1 in central Moravia where the environmental risk is emphasized due to the location of the site not far from drinking water resources. The subsoil at the site is significantly contaminated, mainly by BTEX, mono-chlorobenzene (MCB), chlorinated ethenes (CIE), and drug residuals that are present in the soil and groundwater (citace). The aquifer is shallow and well permeable, with water table at 3$4 \mathrm{~m}$ below ground level.

Dekonta carried out a MIP investigation combined with core sampling in one of the most contaminated areas at the site, contaminated mainly by BTEX and MCB, in order to get information on distribution of the contaminants. Another MIP investigation was performed in the area that was believed to be only slightly contaminated by CIE.

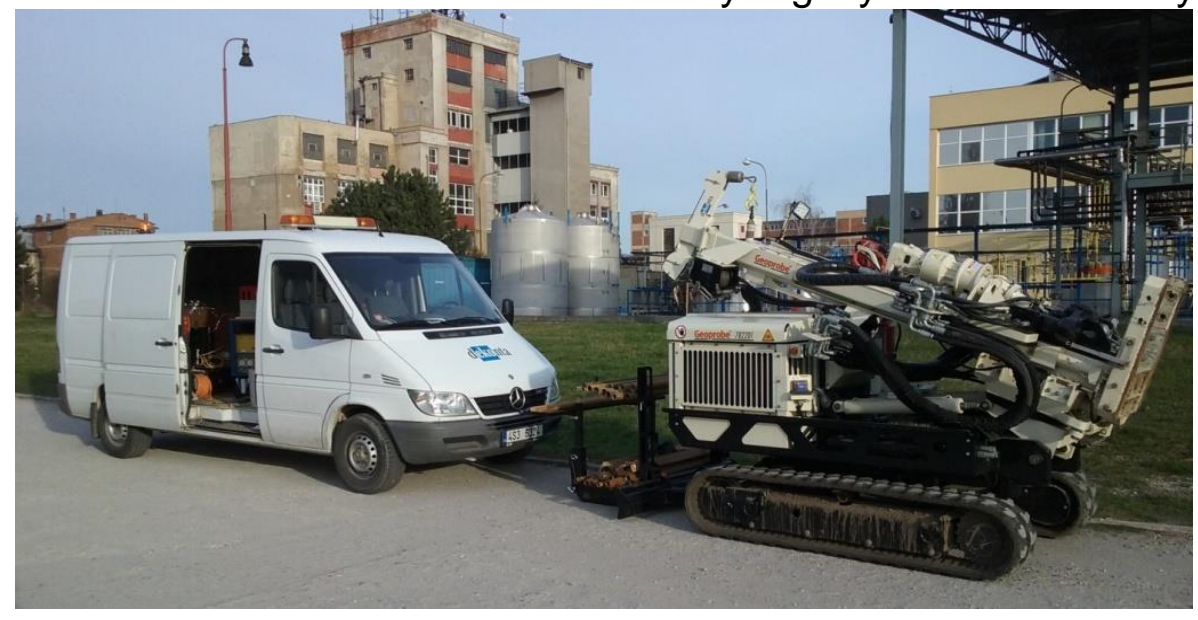

Figure 2: Geoprobe rig 7822DT and MIP van on the Moravian site

Geoprobe 7822 DT and MIP were used for field works (Figure 2). Three detectors were used within the MIP probing - PID, FID, and XSD. A Geoprobe drilling machine was also used for coresoil sampling, in order to correlate the MIP data. The obtained MIP data were processed using the Ejlskov Studio Pro software (provided by the Danish company EJLSKOV), which allows using the raw MIP data to model spatial distribution of the contamination expressed as a particular detector response (see an example in Figure 3 and 4).

The results showed good correlation between the soil samples and the MIP logging. Based on the data obtained, we can state MIP can be used to monitor mixed contamination by BTEX and MCB. Presence of MCB caused similar increase in the PID signal, but it is confirmed by a slight increase of XSD,enabling its distinguishing from BTEX [4]. Following picture shows an example of contamination profile obtained by MIP investigation. 

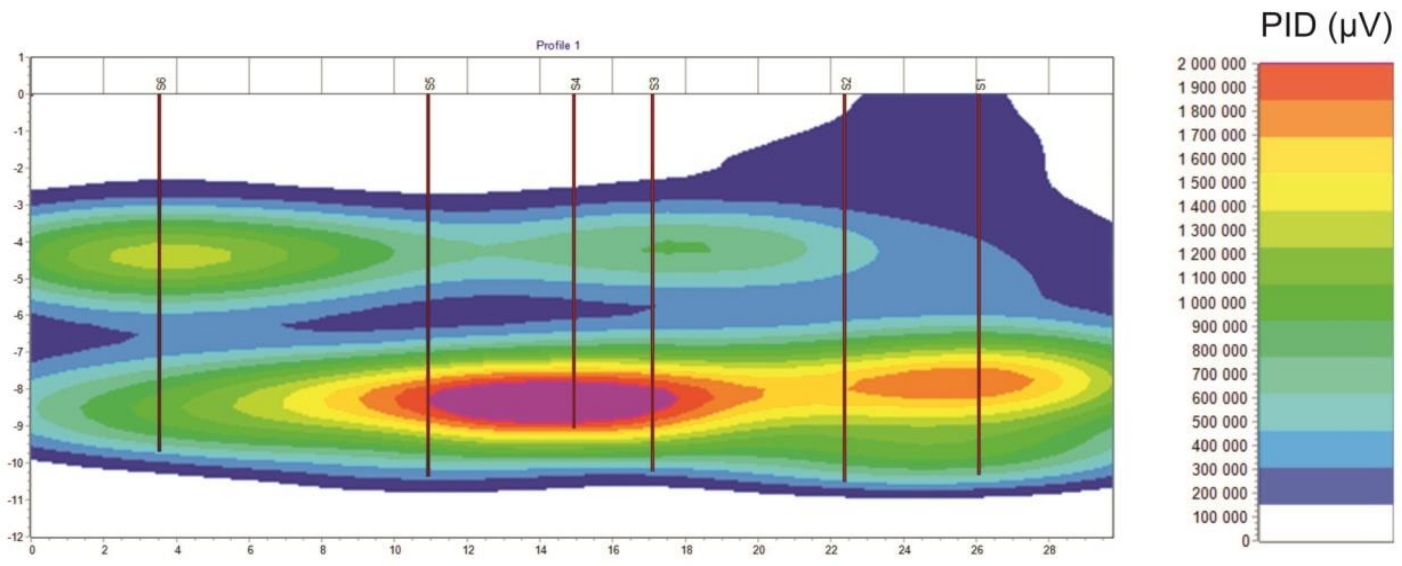

Figure 3: An example of contamination profile (BTEX and MCB) (Ejlskov Studio Pro software)

The MIP investigation of the area contaminated by $\mathrm{CIE}$ discovered strong contamination mainly between $9-11 \mathrm{~m}$ bgs in lower permeable formations. It was an important finding because the depth of all historical monitoring wells within the area reached only to $8 \mathrm{~m}$ bgs. Therefore, the extent of this contamination was unclear before [4]. The pilot test results will help significantly in preparing the final remediation design of the whole contaminated site. We also plan further MIP investigation throughout the course of the remediation activities on the site.

All the works within this site were performed within the research project called PASSES ${ }^{(1)}$, focused on the utilization of innovative monitoring tools for a detailed characterization of contaminated sites.

The pilot test ongoing on the pilot site aims to assess in situ biodegradation potential of the contaminants. Within the framework of the pilot test, different methods of oxygen delivery into the aquifer will be tested, including direct-push injection of 3 different oxygen release compounds using Geoprobe tooling and ISCO agents.

\section{Project \#2}

On the national scene, Dekonta is cooperating with a wide consortium of czech universities and remediation companies. An example of this cooperation is research project Nanobiowat ${ }^{(2)}$ (Environmentally friendly nanotechnologies and biotechnologies in water and soil treatment).

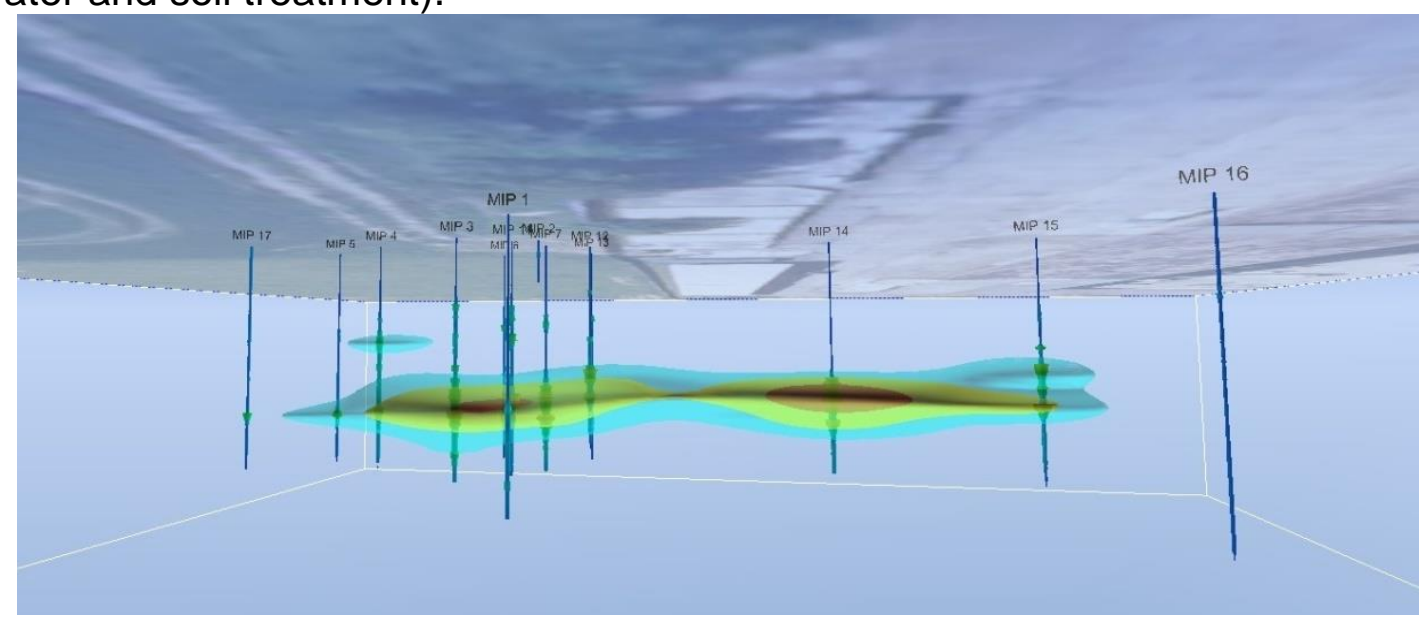

Figure 4: 3D visualisation of CIE contamination plume at Frantiskovy Lazne site (Ejlskov Studio Pro software)

An appropriate and directed injection of remediation agents is crucial for succesful remediation. Especially for agents like iron nanoparticles (nZVI), which has very limited 
migration capabilities [5]. Detailed site characterisation was performed at Site \#2 in Frantiskovy Lazne in cooperation with Aecom CZ. MIP investigation revealed a large contamination plume around $7 \mathrm{~m}$ bgs. (Figure 4). This finding will significantly help to manage further remediation efforts. In this case it is going be mainly direct-push application of $\mathrm{nZVI}$ and whey.

Direct Connection of MIP with Remediation

Dekonta company participates in a number of projects related to improving the MIP technology and its direct connection with remediation activities.

The MIP-IN concept was developed within the FP7 UPSOIL project (EU GA 226956). The innovative MIP-IN device combines (1) detection of pollutants by membrane interface probe (MIP) and (2) a simultaneous correlated injection (IN) during direct push of the device using a drilling machine (Figure 5). Thus, the injected reagent is more targeted towards the real location of the pollution. The first tests of MIP-IN concept were performed within the FP7 UPSOIL project. Results proved that the patented MIP-IN device [6] has potential for simultaneous detection of contaminants and injection of reagents for contaminants removal [7].

Since the end 2013, the MIP-IN device is being further developed and tested within the MIP-IN EUROSTARS-project ${ }^{(3)}$ where VITO (Belgium), Ejlskov (Denmark), Dekonta (Czech Republic) and Ecorem/ABO (Belgium) cooperated. The main goals of the MIPIN EUROSTARS project were to (1) improve the MIP-IN device, (2) validate the MIPIN device in relevant environments and define boundary conditions, and (3) develop an innovative MIP-IN based remediation strategy closely linked with site investigation, based on MIP data interpretation.

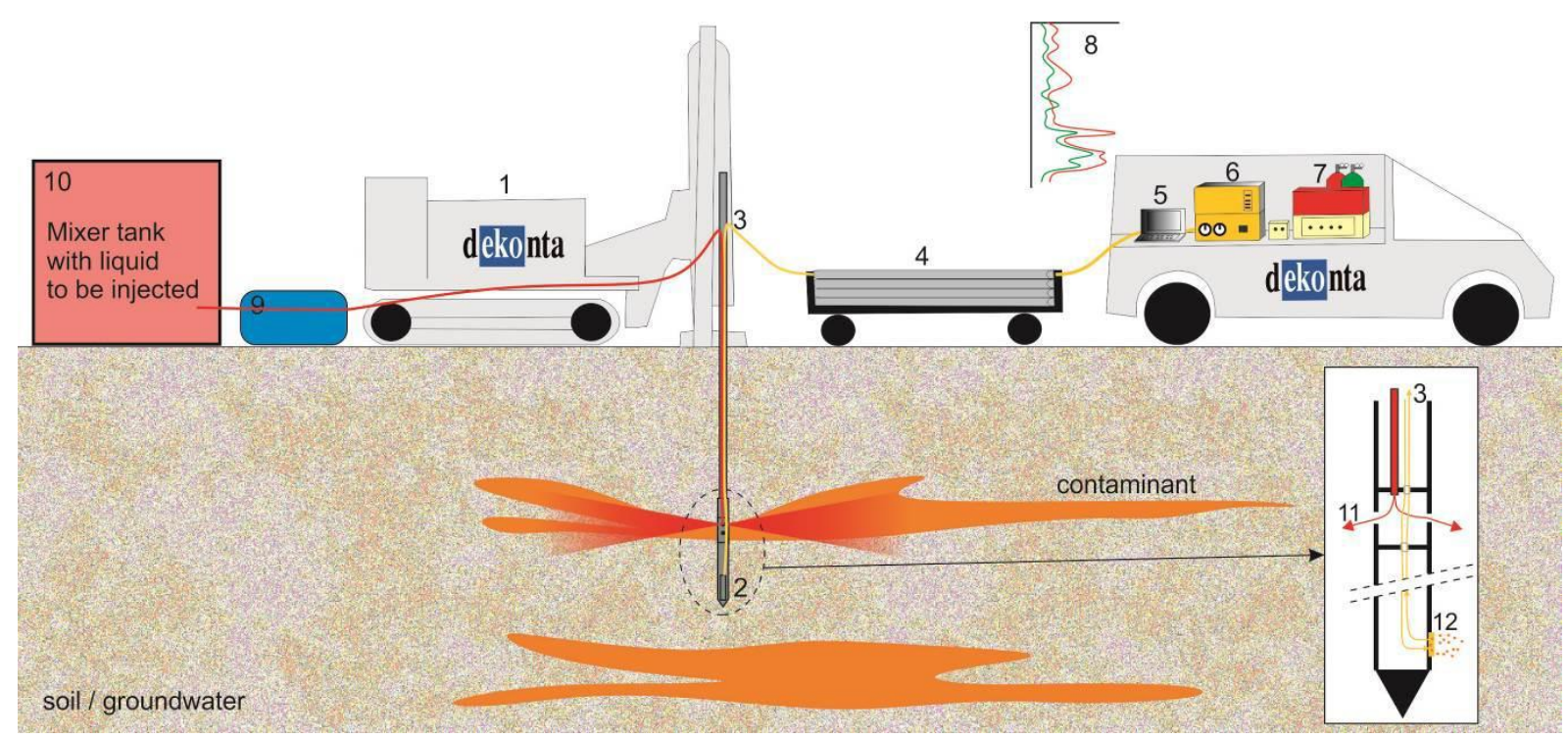

Figure 5: Schematic representation of the MIP-In detection-injection device, 1) drilling machine, 2) MIP-IN probe, 3) tubes with the carrier gas and MIP cables (so-called trunkline), 4) drilling rods holder, 5) PC for MIP data processing, 6) MIP control unit, 7) gas chromatograph with 3 detectors and gas cylinders $\left(\mathrm{N}_{2}, \mathrm{H}_{2}\right), 8$ ) measured signals of MIP probe, 9) high pressure injecting pump, 10) mixer tank with the liquid remediation reagent, 11) reagent injected via jets, 12) contamination entering through the semipermeable membrane

Within the framework of the MIP-IN project, pilot tests of the new remediation technology were carried out in real conditions of contaminated sites. In the end of 2014, one of the four pilot tests of the MIP-IN technology was carried out at contaminated site in Velká Hled'sebe in the Czech Republic [8]. Four reagent injections (nZVI) were 
carried out in connection with detection of volatile organic compounds by means of the MIP probe (Figure 6) .
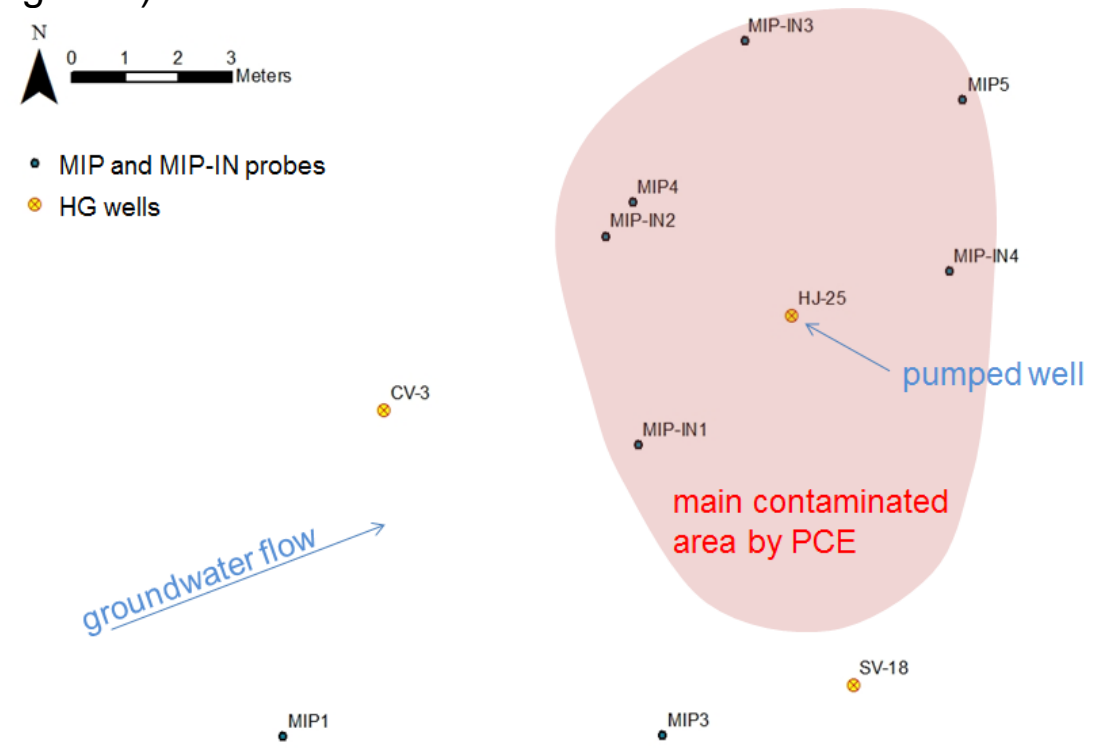

Figure 6: Pilot site for MIP-IN testing in the Czech Republic; (MIP1, MIP3, MIP4 and MIP5) - preinvestigation via MIP probe; (MIP-IN1, MIP-IN2, MIP-IN3 and MIP-IN4)- injections within the pilot tests; (CV3, HJ-25 and SV18) - monitoring hydrogeological wells

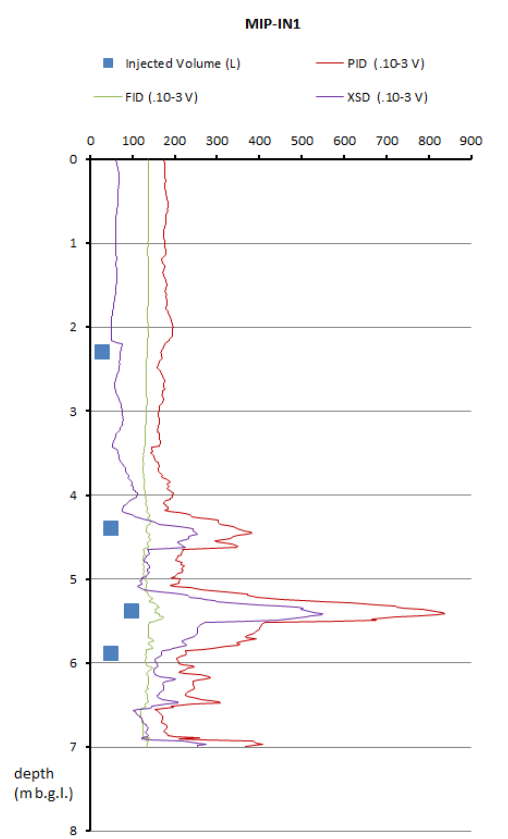

Figure 7: MIP-IN1 probe record including records of injected reagent volume and detector signal
Injections of $\mathrm{nZVI}$ and microZVI suspensions (1200 I, concentration $20 \mathrm{~g} / \mathrm{l}$ ), and of active carbon suspension, by means of the MIP-IN device were successfully tested in the places where contamination presence was detected. The scheme of one MIP-IN probe can be seen on following picture.

Thanks to the pilot tests of the MIP-IN technology, it was possible to define the scope of usability of this technology in the real conditions of the contaminated sites [8].

Another example of connection of MIP technology and remediation is direct-push installation of application wells (Direct-Well system) developed within internal Dekonta research activities. After MIP investigation probe rods are pulled out, then wider rods with expendable tip are driven to a desired depth. The casing (25mm HDPE) is installed via these rods afterwards. The extent of perforation in casing is designed directly on the site according to the results of MIP logs. The space between the casing and the soil is filled with cement-clay mixture.

These direct-wells are suitable for repeatable application of remedial agents under pressure, eventually for groundwater sampling using micropumps. This approach has multiple advantages, mainly quick installation and significant cost reduction. Figure 8 shows results from site in Zdanice contaminated by CIE. Wells will serve for whey application in order to start anaerobic biodegradation processes in the soil. 


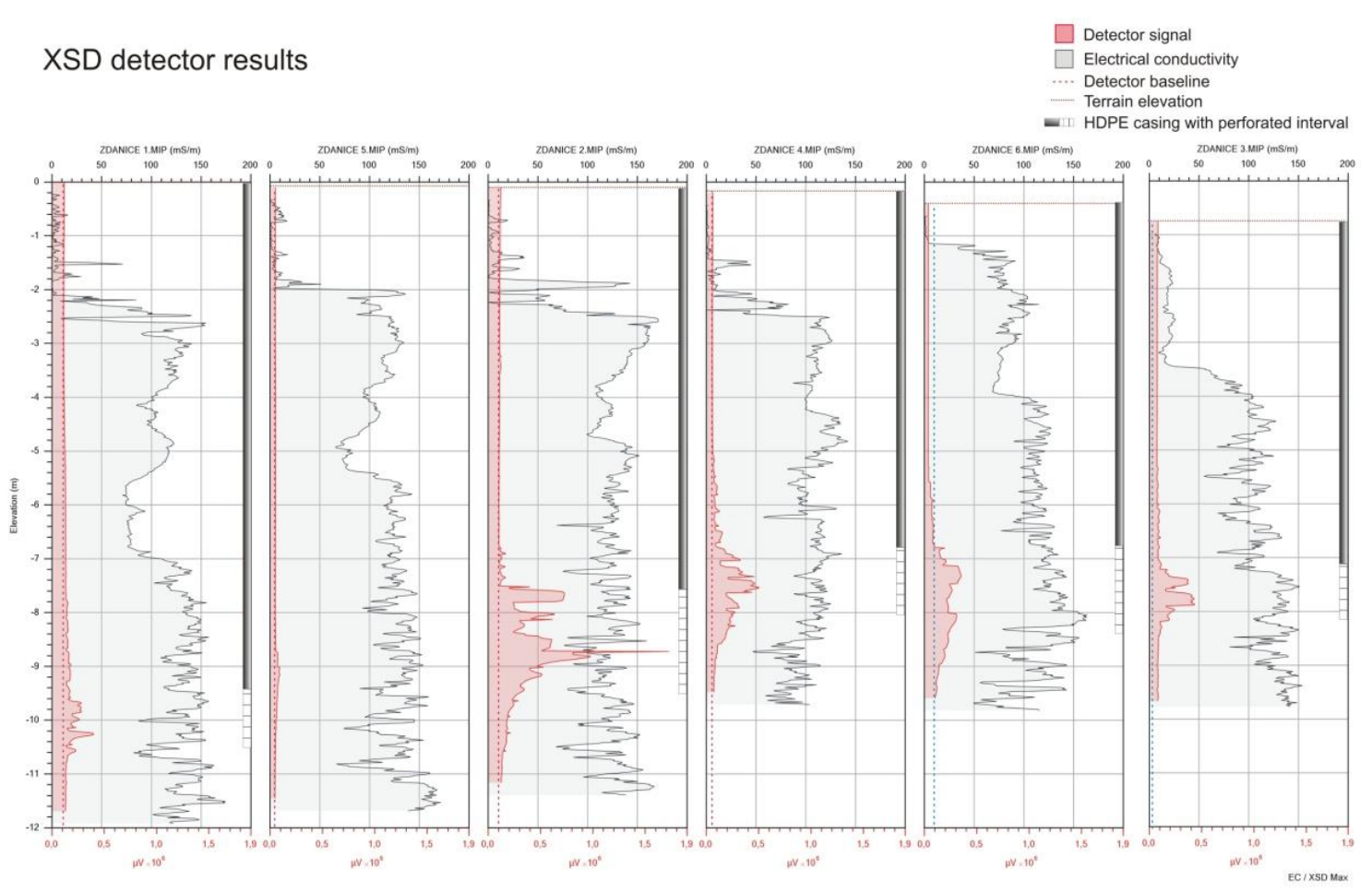

Figure 8: The line of application wells at Zdanice site (Czech Rep.) contaminated by CIE. The perforation of casing is based on XSD signal.

\section{Conclusions}

MIP technology proofed itself as a powerful tool for the contaminated site characterisation. Over the past three years, Dekonta has performed several MIP investigations within its R\&D and commercial activites. As was described in the last part of this article, there is also large potential for improvements and research innovations of this technology.

\section{Acknowledgements}

(1) This project was funded from the Norwegian Financial Mechanism 2009-201,4 and by the Ministry of Education, Youth, and Sports under the Project Contract No. MSMT23681/2015-1

(2) This project Republic No. TE01020218 is supported by the Technology Agency of Czech Republic

(3) This project no. E!8246 was supported by the Ministry of Education, Youth and Sports of the Czech Republic within the programme EUREKA - EUROSTARS. The Eurostars programme is a joint initiative of EUREKA and the European Community.

\section{References}

[1] US EPA, https://clu-in.org/characterization/technologies/hrsc/hrscintro.cfm

[2] McAndrews, B., Heinze, K., DiGuiseppi, W., 2003. Defining TCE plume source areas using the Membrane Interface Probe (MIP). Soil and Sediment Contamination 12(6):799-813

[3] Bronders, J., Van Keer, I., Touchant, K. et al. 2009. Application of the membrane interphase probe (MIP): an evaluation. J Soils Sediments 9: 74.

[4] Dekonta a.s., MIP Logging Used to Investigate Site Contaminated by Mixture of VOCs. 2016. Probing Times Fall 2016. Geoprobe systems. s. 17. 
[5] Shi, Z., Fan, D., Johnson, R. L., Tratnyek, P. G., Nurmic, J.T., Wud, Y., Williams, K. H. 2015. Methods for characterizing the fate and effects of nano zerovalent iron during groundwater remediation. Journal of Contaminant Hydrology. Volume 181: 17-35

[6] Bastiaens, L., and O.P. Stubdrup. 2012. Soil treatment device and use there of for treating contaminated soil and/or groundwater contained therein. Patent WO 2012/123518 A2.

[7] Uyttebroek, M., Stubdrup, P.,O., Hambach, P., Kozubek, P., Kukačka, J., Ulańczyk, R., Otaegi, N., Larsson L., Bastiaens L.: Smart injection technology for efficient in situ remediation: demonstration at a contaminated site in the port of Antwerp. 1st European Symposium on Remediation Technologies and their Integration in Water Management, Barcelona, pp. 215 219., 2012.

[8] Kukačka, J.; Bastiaens, L., De Vos, J., Andersen, B., 2015. MIP-IN: New device for combined detection of pollutants and injection of reagents. Wasteforum No. 4: 232-241. 